\title{
Overexpression of short TRPM8 variant $\alpha$ promotes cell migration and invasion, and decreases starvation-induced apoptosis in prostate cancer LNCaP cells
}

\author{
MOU PENG ${ }^{1 *}$, ZIJUN WANG ${ }^{2 *}$, ZHONGHUA YANG $^{3}$, LIU TAO $^{3}$, \\ QINGLIANG LIU ${ }^{3}$, LU YI $^{1}$ and XINGHUAN WANG ${ }^{3}$
}

\begin{abstract}
Departments of ${ }^{1}$ Urology and ${ }^{2}$ Dermatology, The Second Xiangya Hospital of Central South University, Changsha, Hunan 410011; ${ }^{3}$ Department of Urology, Zhongnan Hospital of Wuhan University, Wuhan, Hubei 430071, P.R. China
\end{abstract}

Received August 16, 2014; Accepted April 27, 2015

DOI: 10.3892/ol.2015.3373

\begin{abstract}
The aim of the present study was to investigate the function of a transient receptor potential melastatin 8 (TRPM8) splice variant, short TRMP8 $\alpha$ (sM8 $\alpha$ ), in the androgen-dependent prostate cancer LNCaP cell line, and to evaluate the potential involvement of the mitogen-activated protein kinase (MAPK) signaling pathway. The coding DNA for sM8 $\alpha$ was cloned and transfected into LNCaP cells to generate cells that overexpress this isoform of TRPM8. Cellular proliferation was determined by performing an MTT assay, and flow cytometry was used to analyze apoptosis and cell cycle distribution. Furthermore, cellular migration and invasion were evaluated using Transwell ${ }^{\circledR}$ migration assays. The subcellular location of recombinant sM8 $\alpha$ was detected by quantum dots-based immunofluorescent imaging, western blotting was performed to examine the expression levels of proteins in the MAPK signaling pathway and reverse transcription-polymerase chain reaction was used to determine the expression of sM $8 \alpha$ mRNA transcripts. The present study demonstrated that sM $8 \alpha$ mRNA was expressed at a low level in the LNCaP, DU145 and PC-3 prostate cancer cell lines. Additionally, the recombinant sM8 $\alpha$ protein was located in the cytoplasm of LNCaP cells and its overexpression significantly reduced starvation-induced
\end{abstract}

Correspondence to: Professor Lu Yi, Department of Urology, The Second Xiangya Hospital of Central South University, 139 Renmin Road, Changsha, Hunan 410011, P.R. China Email: yilu9999@126.com

Professor Xinghuan Wang, Department of Urology, Zhongnan Hospital of Wuhan University, 169 Donghu Road, Wuhan, Hubei 430071, P.R. China

E-mail: urologistwxh@gmail.com

*Contributed equally

Key words: transient receptor potential melastatin 8, variant, alternative splicing, prostate cancer, mitogen-activated protein kinase signaling pathway apoptosis in these cells $(\mathrm{P}<0.05)$, possibly by means of reduced activation of phosphorylated-c-Jun $\mathrm{N}$-terminal kinase (p-JNK). The migration and invasion of the LNCaP cells were markedly enhanced by the overexpression of $\mathrm{sM} 8 \alpha$, possibly via activation of MMP-2. Furthermore, overexpression of sM8 $\alpha$ in LNCaP cells did not alter the expression of full-length TRPM8 and had no effect on cellular proliferation. Overall, the results of the present study indicate that sM8 $\alpha$ may be important in the regulation of prostate cancer cell migration and invasion through the activation of matrix metalloproteinase-2, as well as in the regulation of apoptosis through the activation of p-JNK in the MAPK signaling pathway.

\section{Introduction}

Prostate cancer is the most commonly diagnosed type of carcinoma in men in western industrialized countries (1). Despite initially responding to androgen-deprivation therapy, prostate cancer typically becomes resistant and evolves into recurrent androgen-independent cancer following approximately one year of treatment (2). The mechanism for how this resistance develops is unclear and the development of novel therapeutic strategies for the treatment of androgen-independent prostate cancer are required.

Transient receptor potential melastatin 8 (TRPM8) is a type of $\mathrm{Ca}^{2+}$ permeable cation channel that is potentially associated with tumorigenesis and tumor progression (3). Yang et al (4) transfected TRPM8 into androgen-independent PC-3 prostate cancer cells, and determined that overexpression of TRPM8 inhibits the proliferation and malignant progression of PC-3 cells. A study conducted by Zhang and Barritt (3) revealed that TRPM8 has a vital role in $\mathrm{Ca}^{2+}$ homeostasis in prostate epithelial cells, in addition to being required for cell survival. Therefore, TRPM8 may have an effect on the growth and malignant progression of prostate cancer.

Alternative splice variants contribute to biological complexity and diversity by coding for functional or nonfunctional protein isoforms. TRPM8 isoforms generated by alternative mRNA splicing are expressed in different tissues, such as human lung tissue $(5,6)$ and certain types of prostate cancer (7). The functions of various TRPM8 isoforms 
have previously been described in a number of studies $(8,9)$. For example, short TRPM8 $\alpha(\operatorname{sM} 8 \alpha)$ and short TRPM8 $\beta$ (sM8 $\beta$ ) code for N-terminal fragments of the full-length TRPM8 channel, and regulate TRPM8 activity by stabilizing the closed state of the channel, thus, reducing its activity and cold sensitivity (8). Furthermore, inhibition of TRPM8 activity by sM8 $\beta$, heat or chemical blockers revealed common mechanisms for regulating the single-channel kinetics (9). However, the majority of previous studies reported the functions of short TRPM8 isoforms in human embryonic kidney (HEK) 293 cells. Therefore, research regarding the function of short TRPM8 isoforms in prostate cancer cells is required to elucidate their role in the progression of prostate cancer.

The aim of the present study was to detect the expression of $\mathrm{sM} 8 \alpha$ in various prostate cancer cell lines; to investigate the role of $\mathrm{sM} 8 \alpha$ expression on prostate cancer LNCaP cell line proliferation, apoptosis, migration and invasion; and to examine the involvement of the mitogen activated protein kinase (MAPK) signaling pathway.

\section{Materials and methods}

Cell culture. Human prostate carcinoma LNCaP, DU145 and PC-3 cells were purchased from the American Type Culture Collection (Manassas, VA, USA) and cultured in RPMI 1640 medium (Gibco Life Technologies, Grand Island, NY, USA) containing $10 \%$ fetal bovine serum (FBS; Gibco Life Technologies), $100 \mu \mathrm{g} / \mathrm{ml}$ streptomycin sulfate and $100 \mathrm{U} / \mathrm{ml}$ penicillin G sodium (G4003; Guge Biotech, Wuhan, China). Cells were maintained in a humidified incubator with $5 \% \mathrm{CO}_{2}$ at a temperature of $37^{\circ} \mathrm{C}$.

Reverse transcription-polymerase chain reaction (RT-PCR) for $s M 8 \alpha$. Total RNA was extracted from prostate carcinoma LNCaP, DU145 and PC3 cells using TRIzol reagent (Invitrogen Life Technologies, Carlsbad, CA, USA). A total of $2 \mu \mathrm{g}$ RNA was reverse transcribed (Beijing TransGen Biotech Co., Ltd., Beijing, China) into complementary (c)DNA at $42^{\circ} \mathrm{C}$ using oligo(dT) primers and murine leukemia virus reverse transcriptase (TransScript First-Strand cDNA Synthesis SuperMix; Beijing TransGen Biotech Co., Ltd.), followed by PCR using the 2xTransTaq High Fidelity(HiFi) PCR SuperMix II(-dye) (Beijing TransGen Biotech Co., Ltd). The PCR primers were as follows: Forward, 5'-ATACTCGAGATGGAA GGCACCCAGATCAACCAAAGTGAGAAATGGAACT-3' and reverse, 5'-ATAGAATTCCTAATGATGATGATG ATGATGGCAGACCTCCTCCTGTCCCA-3' for sM8 $\alpha$; and forward, 5'-ACGGATTTGGTCGTATTGGG-3' and reverse, 5'-CGCTCCTGGAAGATGGTGAT-3' for glyceraldehyde phosphate dehydrogenase (GAPDH). For PCR, $2 \mu \mathrm{l}$ cDNA template of the three prostate cancer cell lines were respectively added to a PCR mixture (Beijing TransGen Biotech Co., Ltd.) and then $\mathrm{dd}_{2} \mathrm{O}$ was added up to a final volume of $20 \mu \mathrm{l}$. The DNA amplification conditions were as follows: $95^{\circ} \mathrm{C}$ for $10 \mathrm{~min} ; 35$ cycles of $95^{\circ} \mathrm{C}$ for $30 \mathrm{sec}, 60^{\circ} \mathrm{C}$ for $30 \mathrm{sec}$ and $72^{\circ} \mathrm{C}$ for $1 \mathrm{~min}$; followed by $72^{\circ} \mathrm{C}$ for $10 \mathrm{~min}$. The length of the sM8 $\alpha$ PCR product was $534 \mathrm{bp}$. Furthermore, the PCR product contained two restriction enzyme sites [XhoI (Takara Biotechnology Co., Ltd., Dalian, China) in the
5' extremity and EcoRI (Takara Biotechnology Co., Ltd.) in the $3^{\prime}$ extremity] and a His tag in the 3 ' extremity. GAPDH was used as the housekeeping gene, with the following amplification conditions: $95^{\circ} \mathrm{C}$ for $10 \mathrm{~min} ; 28$ cycles of $95^{\circ} \mathrm{C}$ for $30 \mathrm{sec}, 56^{\circ} \mathrm{C}$ for $30 \mathrm{sec}$ and $72^{\circ} \mathrm{C}$ for $30 \mathrm{sec}$; followed by $72^{\circ} \mathrm{C}$ for $10 \mathrm{~min}$. The length of the GAPDH PCR product was 214 bp. All PCR products were analyzed by gel electrophoresis and DNA sequencing.

Plasmid construction. A pcDNA3.1(-) eukaryotic expression clone vector (Invitrogen Life Technologies) was used in the present study. The PCR product contained the gene of interest, a His tag and XhoI and EcoRI restriction enzyme sites. The plasmid and PCR product were digested by XhoI and EcoRI prior to isolation by DNA gel extraction (Axygen, Hangzhou, China). Then, the digested plasmid and PCR product sequences were linked using T4 DNA ligase (Thermo Fisher Scientific Inc., Beijing, China), and transformed into DH5 $\alpha$ cells (Biovector NTCC, Inc., Beijing, China) for synthesis of the pcDNA3.1(-)-sM8 $\alpha$-His plasmid. The plasmid was sequenced and found to contain no mutations.

Cell transfection for stable cell clone. LNCaP cells were plated into a six-well plate at a density of $10^{5}$ cells/well, and transfected at $\sim 90-95 \%$ confluence with the recombinant pcDNA3.1(-)-sM8 $\alpha$-His and pcDNA3.1(-) negative control (NC) plasmids using $10 \mu$ l Lipofectamine 2000 (Invitrogen Life Technologies, Carlsbad, CA, USA), according to the manufacturer's instructions. Stably transfected clones were selected using G418 (Sigma-Aldrich, St Louis, MO, USA) at a concentration of $700 \mu \mathrm{g} / \mathrm{ml}$. Colonies were identified using RT-PCR and western blot analysis. In the present study, LNCaP-sM8 $\alpha$ cells refer to LNCaP cells transfected with and overexpressing His-tagged sM8 $\alpha$, and LNCaP-NC cells refer to $\mathrm{LNCaP}$ cells transfected with empty vector.

Cell proliferation. Cell proliferation was detected by performing a 3-(4,5-dimethylthiazol-2-yl)-2, 5-diphenyltetrazolium bromide (MTT) assay. Cells were seeded in 96-well plates at a density of $1 \times 10^{3}$ cells per well and were incubated for 1-5 days. Subsequently, $20 \mu \mathrm{l}$ MTT (concentration, $5 \mathrm{mg} / \mathrm{ml}$ ) was added to each well and the plate was incubated for $4 \mathrm{~h}$. The liquid was removed from each well and replaced with $150 \mu 1$ DMSO. The optical density (OD) value of each well was measured at a wavelength of $570 \mathrm{~nm}$ using an MD2 Microplate Reader (Molecular Devices, Sunnyvale, CA, USA).

Quantum dots (QDs)-based immunofluorescent imaging. LNCaP-sM8 $\alpha$ and LNCap-NC cells were plated on 12-mm coverslips and incubated overnight, prior to being fixed with $1 \%$ neutral formaldehyde at room temperature for $10 \mathrm{~min}$. To prepare samples for QD-based immunofluorescent imaging, cells were first blocked with $2 \% \mathrm{w} / \mathrm{v}$ bovine serum albumin (BSA) for $30 \mathrm{~min}$ at $37^{\circ} \mathrm{C}$ and then incubated with the primary anti-His-tag antibody for $4 \mathrm{~h}$ at $37^{\circ} \mathrm{C}$. The cells were incubated with secondary antibody (QDs-605) for $2 \mathrm{~h}$ at $37^{\circ} \mathrm{C}$ following an a second blocking step with $2 \%$ BSA for $30 \mathrm{~min}$. Cells were subsequently incubated with DAPI $(5 \mu \mathrm{g} / \mathrm{ml})$ for $3 \mathrm{~min}$ at room temperature to stain the nuclei. The samples were washed with Tris-buffered saline and examined under 
an Olympus BX51 fluorescence microscope equipped with an Olympus DP72 camera (Olympus Corporation, Tokyo, Japan). The QDs-605 and DAPI were excited by ultraviolet light (wavelength, $388 \mathrm{~nm}$ ) (10).

Flow cytometry. LNCaP-NC and LNCaP-sM8 $\alpha$ cells were prepared for cell cycle and apoptosis assays (kit from Kaiji Biotechnology Co., Ltd., Nanjing, China), according to the manufacturer's instructions. Briefly, the cells were fixed with $70 \%$ ethanol overnight at a temperature of $4^{\circ} \mathrm{C}$, washed with phosphate-buffered saline and stained with propidium iodide. Subsequently, the cell cycle stage was determined and analyzed by performing flow cytometry. To evaluate apoptosis, cells were seeded in complete culture medium for $24 \mathrm{~h}$ and then incubated in culture medium containing $1 \%$ fetal bovine serum (FBS) for an additional $48 \mathrm{~h}$. The percentage of apoptotic cells were determined using flow cytometry, according to the manufacturer's instructions (Kaiji Biotechnology Co., Ltd.). Details of the flow cytometry experiments have been previously described (4).

Cell migration and invasion assays. To assay cell migration, $2 \times 10^{4}$ cells suspended in $200 \mu 1$ RPMI-1640 medium without FBS were seeded onto the fibronectin-coated polycarbonate membrane of a Transwell ${ }^{\circledR}$ insert [Becton Dickinson Medical Devices (Shanghai) Co., Ltd., Shanghai, China]. A volume of $600 \mu 1$ RPMI-1640 with 10\% FBS was added as a chemoattractant in the lower chamber. Following incubation for $12 \mathrm{~h}$ at $37^{\circ} \mathrm{C}$ in a $5 \% \mathrm{CO}_{2}$ atmosphere, the Transwell insert was washed with PBS and the cells on the top surface of the insert were removed with a cotton swab. Cells adhering to the lower surface were fixed with methanol for $10 \mathrm{~min}$, stained with $0.1 \%$ Giemsa solution for $10 \mathrm{~min}$, thrice-washed with PBS and finally air-dried. Five predetermined fields (magnification, $\mathrm{x} 200$ ) were counted using a fluorescence microscope (BX51; Olympus Corporation, Tokyo, Japan). All assays were independently repeated in triplicate. The cell invasion assay procedure was similar to that for cell migration, except the Transwell membranes were pre-coated with $50 \mu \mathrm{g} / \mu \mathrm{l}$ Matrigel $^{\circledR}$ (BD Biosciences, Franklin Lakes, NJ, USA) and the cells were incubated for $48 \mathrm{~h}$ at $37^{\circ} \mathrm{C}$ in a $5 \% \mathrm{CO}_{2}$ atmosphere. Cells adhering to the lower surface were counted in the same manner as for the cell migration assay.

Western blot analysis. Protein expression levels of the sM8 $\alpha$-His fusion protein, TRPM8, matrix metalloproteinase (MMP)-2, MMP-9, MAPK signaling pathway proteins [p38, c-Jun N-terminal kinase (JNK) and extracellular signal-regulated kinase 1/2 (ERK1/2)] and GAPDH were assayed using western blot analysis. Equal quantities of protein $(30 \mu \mathrm{g})$ were separated by $10 \%$ sodium dodecyl sulfate-polyacrylamide gel electrophoresis and then transferred to electrochemiluminescence nitrocellulose membranes (GE Healthcare Life Sciences, Piscataway, NJ, USA). Primary antibodies against human TRPM8 (rabbit polyclonal; catalog no. ACC-049; Alomone Labs, Jerusalem, Israel; 1:500 dilution), MMP-2 (rabbit monoclonal IgG; catalog no. 13132; Cell Signaling Technology, Inc., Danvers, MA, USA; 1:1,000 dilution), MMP-9 (rabbit monoclonal IgG; catalog no. 13667, Cell Signaling Technology, Inc.;
1:1,000 dilution), GAPDH (rabbit polyclonal IgG; catalog no. sc-25778, Santa Cruz Biotechnology, Inc., Dallas, TX, USA; 1:1000 dilution), MAPK family [rabbit anti-p38, ERK1/2 (p44/42) and JNK (catalog no. 9926), and phospho (p-)p38, p-ERK1/2 and p-JNK (catalog no. 9910); Cell Signaling Technology, Inc.] and His-tag (mouse monoclonal IgG2a; catalog no. D291-3, Medical \& Biological Laboratories Co., Ltd., Nagoya, Japan) were applied overnight at $4^{\circ} \mathrm{C}$. Polyclonal goat anti-rabbit (catalog no. sc-2005; 1:5,000 dilution) and goat anti-mouse (catalog no. sc-2004; 1:5,000 dilution) IgG horseradish peroxidase-conjugated (Santa Cruz Biotechnology, Inc.) secondary antibodies were then applied for $2 \mathrm{~h}$ at $37^{\circ} \mathrm{C}$. Protein bands were visualized using an ECL Western Blotting kit (Guge Biotech, Wuhan, China). The results of western blot were analyzed by Image-Pro Plus 6.0 (Media Cybernetics, Inc., Rockville, MD, USA) and the integrated optical density values were normalized to GAPDH, which was the loading control. The procedure was performed as previously described (4) and each experiment was repeated three times with similar results.

Statistical analysis. SPSS software for Windows (version 13.0; SPSS, Inc., Chicago, IL, USA) was used to perform all statistical analyses. All data are presented as the mean \pm standard error of the mean. Statistical analyses were performed using the unpaired t-test, with $\mathrm{P}<0.05$ considered to indicate a statistically significant difference.

\section{Results}

Expression of sM8a in three prostate cancer cell lines and its effect on cell proliferation. sM8 $\alpha$ mRNA expression levels were investigated in three prostate cancer cell lines (LNCaP, DU145 and PC-3) using RT-PCR, revealing that the expression level of sM8 $\alpha$ was low in all three lines. Following stable transfection of sM8 $\alpha$ into LNCaP cells, sM8 $\alpha$ mRNA expression levels were detected in LNCaP, LNCaP-NC and LNCaP-sM8 $\alpha$ cells. As expected, expression of sM8 $\alpha$ in the LNCaP-sM8 $\alpha$ cells was high compared with the LNCaP-NC cells (Fig. 1A). Cell growth curves for the LNCaP-sM8 $\alpha$ and LNCaP-NC cells were generated using OD data obtained on days 1-5. No significant difference in cell proliferation was identified between the LNCaP-sM8 $\alpha$ and LNCaP-NC cells ( $\mathrm{P}>0.05$; Fig. 1B).

Subcellular location of the sM8 $\alpha$-His fusion protein in stably transfected LNCaP cells. QD-based immunofluorescent imaging revealed clearly observable red immunofluorescence in the majority of LNCaP-sM8 $\alpha$ cells examined, indicating expression of the sM8 $\alpha$-His fusion protein. Furthermore the sM8 $\alpha$-His fusion protein was visualized in cytoplasm. By contrast, immunostaining did not detect a red immunofluorescence signal for the sM8 $\alpha$-His fusion protein in LNCaP-NC cells; instead, only DAPI-stained blue fluorescent nuclei were observed (Fig. 1C).

Flow cytometry analysis of cell cycle distribution and apoptosis. The results demonstrated that there were no significant differences in the cell cycle between LNCaP-NC and LNCaP-sM8 $\alpha$ cells (Fig. 2A and B). The effect of the 
A

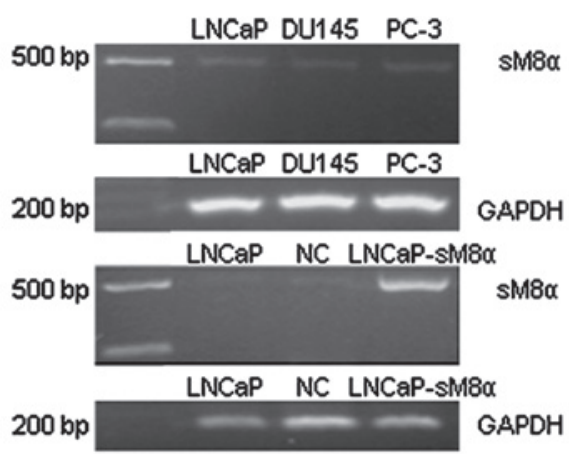

C

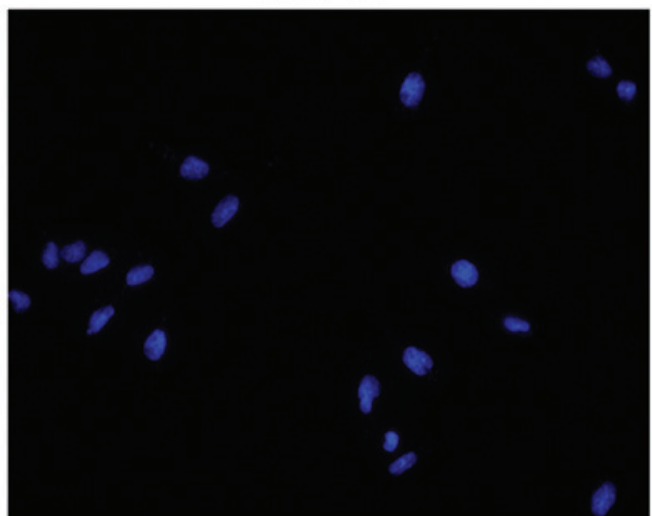

B

Cell growth curve

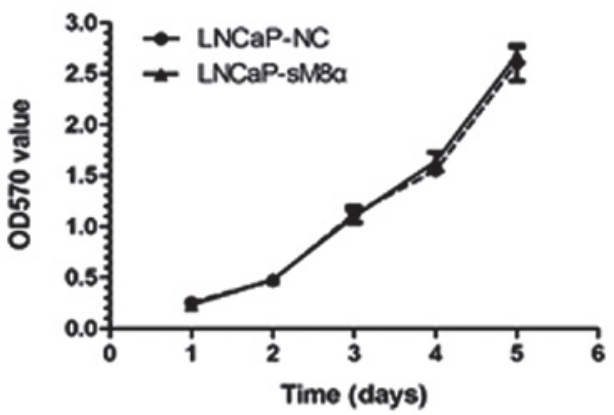

LNCaP-sM8 $\alpha$

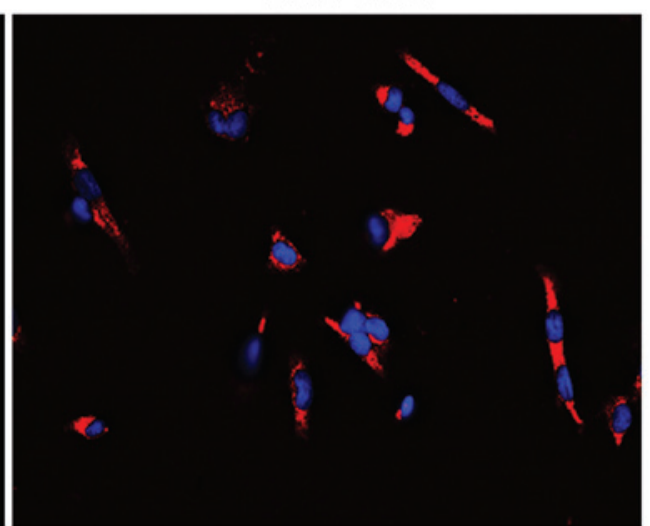

Figure 1. (A) sM8 $\alpha$ and GAPDH mRNA expression levels were analyzed in LNCaP, DU145 and PC-3 cells. sM8 $\alpha$ and GAPDH mRNA expression were also analyzed in LNCaP-sM8 $\alpha$ and LNCaP-NC cells. (B) A cell growth curve for sM8 $\alpha$-overexpressing and control cells. (C) Quantum dots-based immunofluorescent imaging for sM8 $\alpha$-His fusion protein (red) and nuclear staining by DAPI (blue). sM8 $\alpha$, short transient receptor potential melastatin $8 \alpha$; GAPDH, glyceraldehyde phosphate dehydrogenase; NC, negative control; OD, optical density.

A

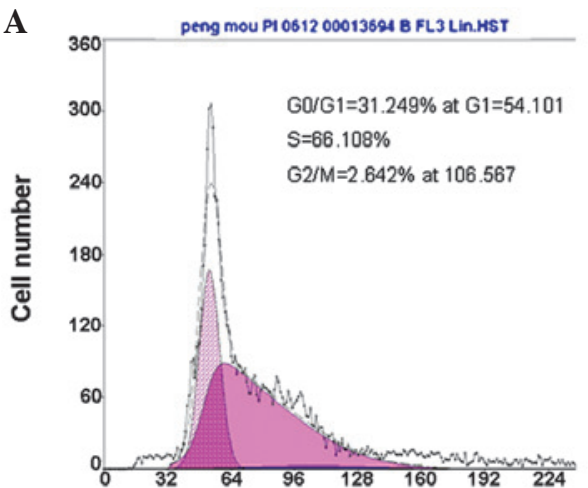

[A] FL1 LogFL 3 Log - ADC

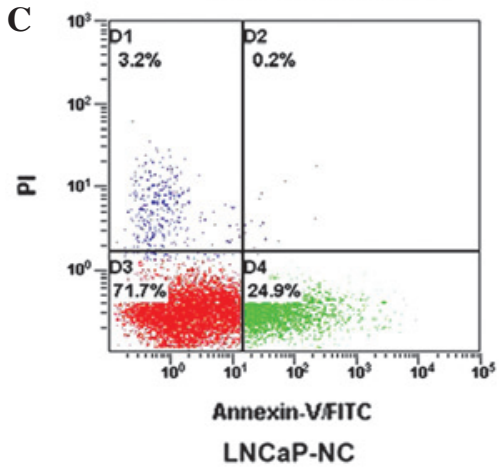

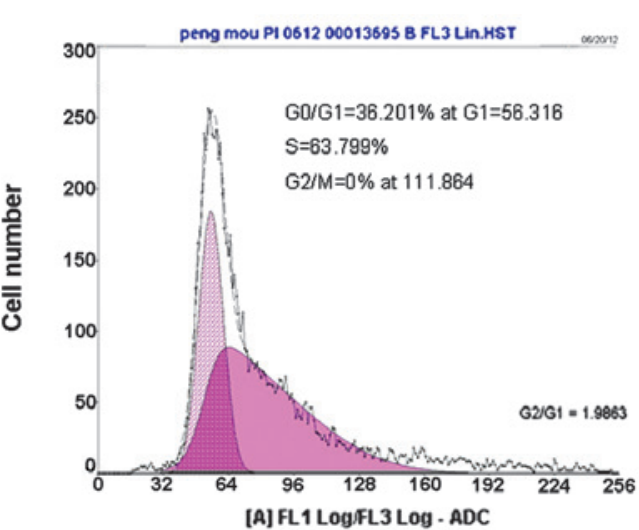

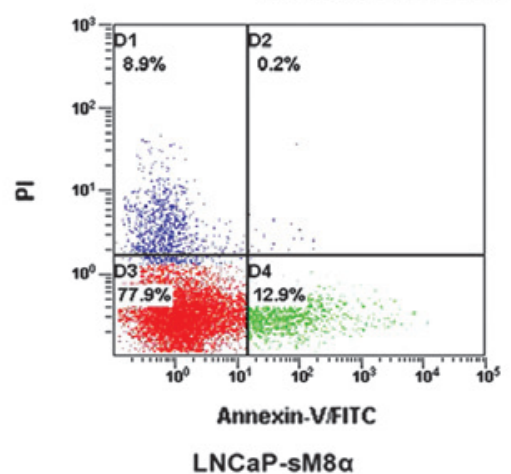

B

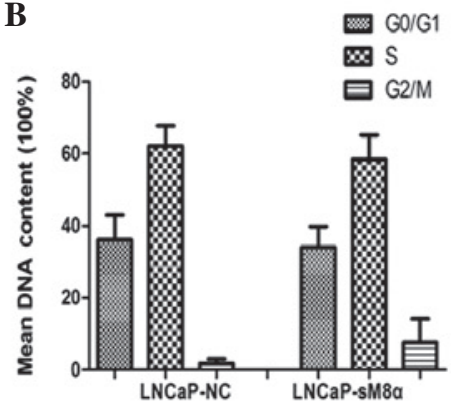

D

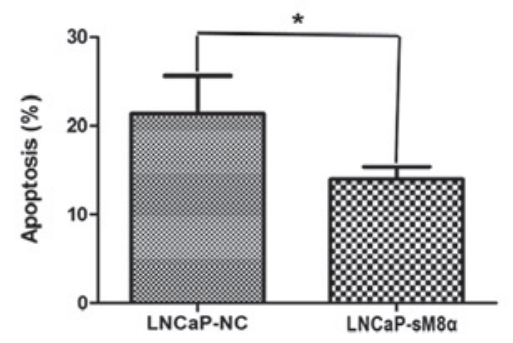

Figure 2. Cell cycle distribution and starvation-induced apoptosis of LNCaP-NC and LNCaP-sM8 $\alpha$ cells. (A and B) Cells stained with PI were analyzed to determine the progression through the cell cycle using flow cytometry. (C and D) Following treatment with RPMI 1640 supplemented with $1 \%$ fetal bovine serum for $48 \mathrm{~h}$, the cells were incubated with FITC-conjugated Annexin V and PI at room temperature for 5 min in the dark. The percentages of cell apoptosis were then determined using flow cytometry. Data are presented as the mean \pm standard deviation of three independent experiments."P $<0.05$. NC, negative control; sM8 $\alpha$, short transient receptor potential melastatin $8 \alpha$; PI, propidium iodide; FITC, fluorescein isothiocyanate. 
A

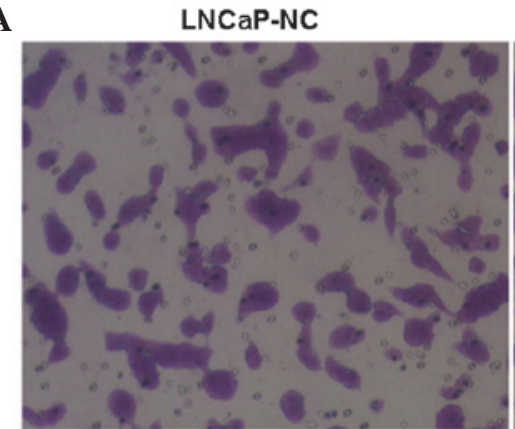

C

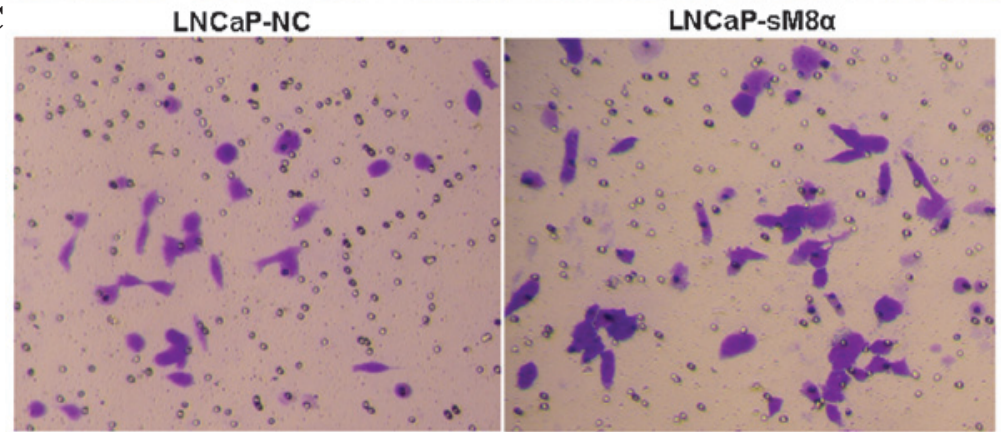

LNCaP-sM8 $\alpha$

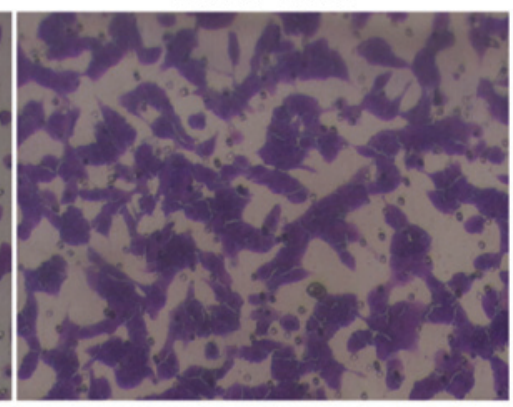

D

B
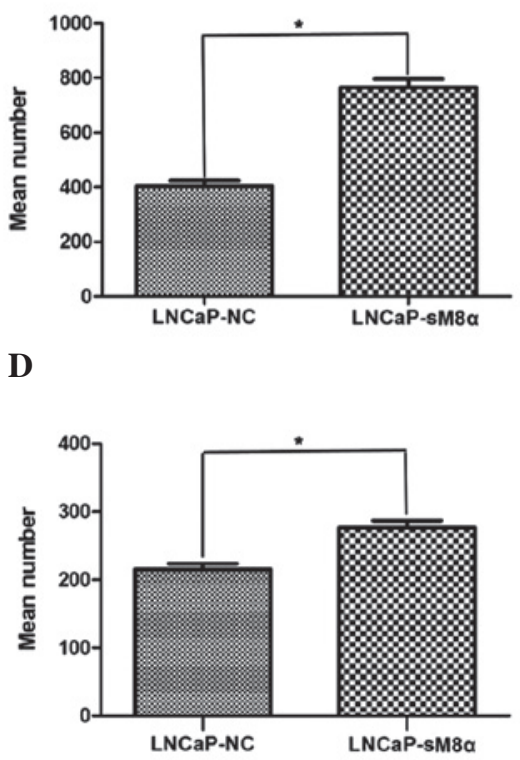

Figure 3. Upregulation of sM8 $\alpha$ increases migration and invasion of LNCaP cells. (A and B) Upregulation of sM8 $\alpha$ significantly increases LNCaP cell migration in vitro. (C and D) Overexpression of sM8 $\alpha$ significantly increased LNCaP cell invasion in vitro (magnification, $\mathrm{x} 200$ ). Data are presented as means \pm standard deviation for three independent experiments. $\mathrm{P}<0.05$. NC, negative control; sM8 $\alpha$, short transient receptor potential melastatin $8 \alpha$.

sM8 $\alpha$-His fusion protein on the apoptosis of transfected LNCaP cells was also investigated. Following incubation in RPMI 1640 with 1\% FBS for 48 h, sM8 $\alpha$-His fusion protein was identified to exhibit a significant antiapoptotic effect on LNCaP cells. The percentage of apoptotic LNCaP-sM8 $\alpha$ cells was significantly lower than the proportion of apoptotic LNCaP-NC cells (13.93 \pm 0.84 vs. $21.33 \pm 2.47 \%$; $\mathrm{P}<0.05$; Fig. 2C and D).

Enhanced cell migration and invasion in LNCaP-sM8a cells. High rates of cell migration and invasiveness are characteristics of cancer cells, indicating and contributing to malignancy (11). Therefore, cell migration and invasion are common targets of anticancer treatment strategies. Cell counts of the lower surfaces of the Transwell membranes revealed that the migration of LNCaP-sM8 $\alpha$ cells was significantly increased following $12 \mathrm{~h}$ of incubation when compared with LNCaP-NC cells $(\mathrm{P}<0.05$; Fig. $3 \mathrm{~A}$ and $\mathrm{B})$. Subsequent analysis of the cell count data revealed a significant increase in the invasiveness of LNCaP-sM8 $\alpha$ cells compared with LNCaP-NC cells $(\mathrm{P}<0.05$; Fig. 3C and D). Furthermore, western blotting (Fig. 4) of MMP-2 and MMP-9 indicated that overexpression of sM8 $\alpha$ may increase the migration and invasion in $\mathrm{LNCaP}$ cells via significantly increasing the proportion of active, $64-\mathrm{kDa}$ MMP-2 (Fig. 4A, G and H).

p-JNK/MAPK signaling pathway may be important in antiapoptosis. The MAPK signaling pathway is an important signaling pathway, and is involved in the regulation of cell proliferation and apoptosis (12). Therefore, western blotting was performed to detect possible changes in the expression levels of proteins in this signaling pathway. The expression of p-JNK was significantly reduced compared in LNCaP-sM8 $\alpha$ cells with the control $(\mathrm{P}<0.05$; Fig. $4 \mathrm{~A}$ and $\mathrm{C})$, indicating its possible involvement in the antiapoptotic mechanism of $\mathrm{mS} 8 \alpha$. By contrast, $\mathrm{p} 38$ and ERK1/2 expression exhibited no significant change in expression between LNCaP-sM8 $\alpha$ cells and control cells $(\mathrm{P}<0.05$; Fig. 4A, D and F). Changes in the expression level of full-length TRPM8, which is known to be regulated by $\mathrm{sM} 8 \alpha$ and $\mathrm{sM} 8 \beta$ in terms of cold sensitivity and channel activity in HEK293 cells (9), were not observed in the LNCaP-sM8 $\alpha$ and LNCaP-NC cells (P>0.05; Fig. 4A and E).

\section{Discussion}

Alternative splice variants are the product of mRNA post-transcriptional processing and have an important effect on the biological behavior of cancer cells. Thus far, the splice variants of numerous ion channels, including TRP channels, have been described $(13,14)$. The role of these TRP-channel splice variants in biological behavior is becoming increasingly clear; however, at present, there is little information regarding the functions of TRPM8 isoforms in the field of carcinogenesis and cancer progression. Therefore, the role of $\mathrm{sM} 8 \alpha$, which encodes the $\mathrm{N}$-terminal fragment of TRPM8, was investigated in prostate cancer cells in the present study.

At least three short TRPM8 isoforms, each exhibiting their own pathophysiological functions, have been reported in previous studies. Sabnis et al (6) reported that the full-length TRPM8 transcript was absent in human lung epithelial cells and identified a novel truncated TRPM8 variant that was selectively expressed as a modulator of respiratory physiology in cold air. Furthermore, Bidaux et al (8) reported the following two novel short splice variants of TRPM8, which were cloned from prostate cancer cells, using a model of HEK 293 cells: sM8 $\alpha$ and sM8 $\beta$. The results demonstrated 
A
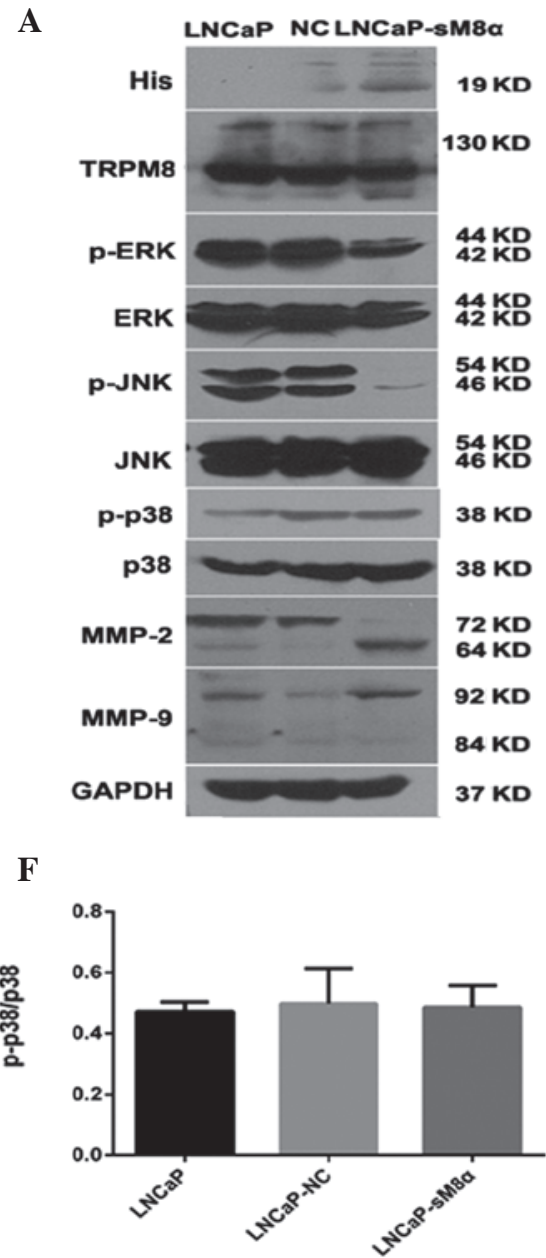

B
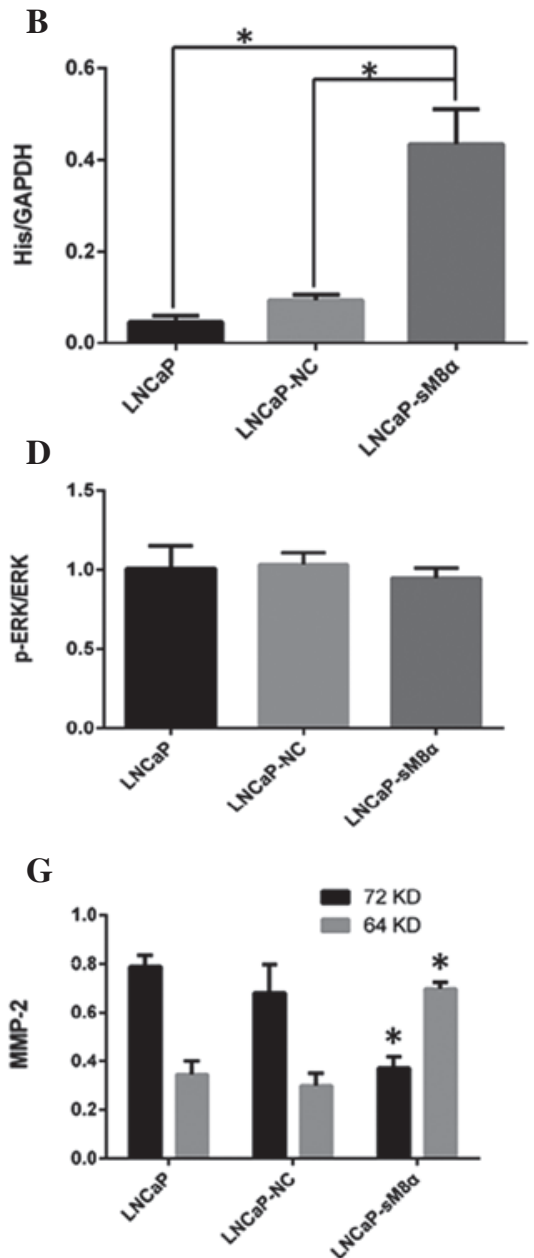

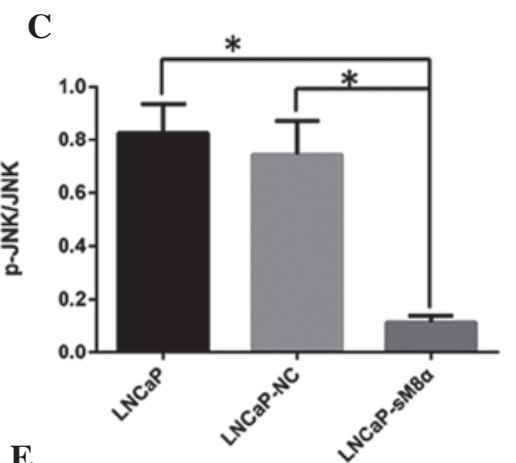

$\mathbf{E}$
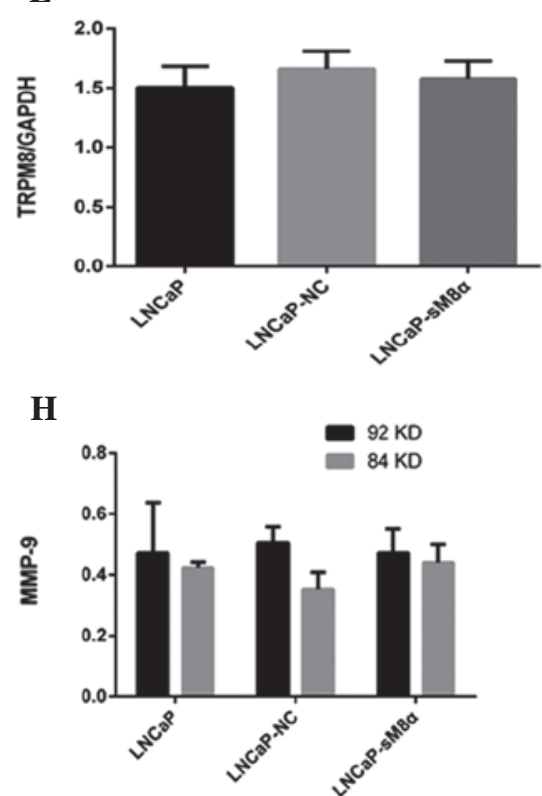

Figure 4. (A) Western blot analysis indicating that overexpression sM8 $\alpha$-His fusion protein reduced the expression of p-JNK and increased the expression of active MMP-2 (64 kDa) in LNCaP cells. GAPDH was used as the inner control. (B) sM8 $\alpha$-His fusion protein was significantly overexpressed in LNCaP-sM8 $\alpha$ cells. (C) The expression of p-JNK protein was significantly reduced in LNCaP-sM8 $\alpha$ cells compared with LNCaP and LNCaP-NC cells. However, there were no significant changes in the expression of (D) p-ERK1/2 and (F) p-p38 or (E) full-length TRPM8 protein. (G) MMP-2 was activated, as indicated by upregulation of 64-kDa MMP-2 and downregulation of 72-kDa MMP-2 in LNCaP-sM8 $\alpha$ cells. (H) There were no remarkable differences in the expression of MMP-9 in LNCaP, LNCaP-NC and LNCaP-sM8 $\alpha$ cells. ${ }^{*} \mathrm{P}<0.05$. NC, negative control; sM8 $\alpha$, short transient receptor potential melastatin $8 \alpha$; TRPM8, transient receptor potential melastatin 8; p-ERK, phosphorylated-extracellular signal-regulated kinase 1/2; p-JNK, phosphorylated-c-Jun N-terminal kinase; MMP, matrix metalloproteinase; GAPDH, glyceraldehyde phosphate dehydrogenase.

that the two variants were in a closed configuration with the C-terminal tail of the full-length TRPM8 channel, resulting in stabilization of its closed state, and reducing its cold sensitivity and activity. Additionally, Fernández et al (9) identified that, in addition to increased temperature, or treatment with BCTC or clotrimazole, short sM8-6 isoforms of TRPM8 inhibited the channel. The present study investigated $\mathrm{sM} 8 \alpha$ by generating an expression vector containing only the sM8 $\alpha$ coding sequence and a His-tag, encoding a protein of $19 \mathrm{kDa}$. This was different to the sM8 $\alpha$ splice variant reported by Bidaux et al (8), which encoded two protein isoforms of 6 and $18 \mathrm{kDa}$. This discrepancy may result from the existence of a regulatory sequence in the sM8 $\alpha$ plasmid allowing for further splicing. Subsequent QDs-based immunofluorescent imaging revealed the $\mathrm{sM} 8 \alpha$-His fusion protein located in the cytoplasm of the LNCaP cells.

Apoptosis is a fundamental cellular process regulated by precise gene expression (15). In the present study, LNCaP cell apoptosis was induced by starvation and it was identified that overexpression of sM8 $\alpha$ could significantly reduce the percentage of apoptotic LNCaP cells. MMP-2 and MMP-9 are key proteins in cancer progression, and are involved in the initial breakdown of collagen and basement membrane components during tumor growth and invasion (16). The present study used Transwell chambers to simulate the basement membrane for migration and invasion, and the results indicated that overexpression of sM8 $\alpha$, through activation of MMP-2, may increase the migration and invasion of LNCaP cells.

The activity of TRPM8 is regulated by a number of cellular signaling pathways, most notably by phosphoinositides and the activation of phospholipase $\mathrm{C}(17)$. However, the cellular signaling pathways regulated by $\mathrm{sM} 8 \alpha$ in prostate cancer cells are unclear. The three major MAPKs (p38, JNK, and ERK1/2) are signal transducers involved in a broad range of prostate cancer cell functions, including survival, apoptosis and cell differentiation (18). The present study demonstrated a role for the MAPK signaling pathway in the regulation of sM8 $\alpha$. Although the ERK1/2 and p38 signaling pathways did not appear to be regulated by $\operatorname{sM} 8 \alpha$, significantly reduced activation of $\mathrm{p}$-JNK was identified in LNCaP-sM8 $\alpha$ cells. We 
hypothesize that this may be associated with the reduction of LNCaP-sM8 $\alpha$ cell apoptosis.

The physiological roles of short TRPM8 isoforms require further investigation. For example, the functions of short TRPM8 isoforms are unclear in prostate cancer cells not expressing full-length TRPM8 and it remains to be elucidated whether short TRPM8 isoforms can influence the release of $\mathrm{Ca}^{2+}$ from the endoplasmic reticulum. Furthermore, additional evidence is required to determine if short TRPM8 isoforms negatively regulate TRPM8 in non-cancerous prostate cells (including normal prostate and benign hyperplasia of the prostate tissue), as well as in cancerous prostate cells.

In conclusion, the present study demonstrated that LNCaP cells express low levels of sM8 $\alpha$. The results indicate that overexpression of sM8 $\alpha$ has no detectable affect on the proliferation of LNCaP cells; however, sM8 $\alpha$ overexpression did appear to increase cell migration and invasion by activation of MMP-2. Furthermore, the antiapoptotic effect of $\mathrm{sM} 8 \alpha$ may be regulated by activation of $\mathrm{p}-\mathrm{JNK}$ in $\mathrm{LNCaP}$ cells. Additional studies of short TRPM8 isoforms should be performed to gain a greater understanding of the functions of the TRPM8 channel.

\section{Acknowledgements}

The present study was supported by the Program of Chinese National Natural Science Fund (grant no. 81172734).

\section{References}

1. Spies E, Reichardt W, Alvarez G, et al: An artificial PAP gene breaks self-tolerance and promotes tumor regression in the TRAMP model for prostate carcinoma. Mol Ther 20: $555-564,2012$.

2. Feldman BJ and Feldman D: The development of androgen-independent prostate cancer. Nat Rev Cancer 1: 34-45, 2001.

3. Zhang L and Barritt GJ: Evidence that TRPM8 is an androgen-dependent $\mathrm{Ca}^{2+}$ channel required for the survival of prostate cancer cells. Cancer Res 64: 8365-8373, 2004.

4. Yang ZH, Wang XH, Wang HP and Hu LQ: Effects of TRPM8 on the proliferation and motility of prostate cancer PC-3 cells. Asian J Androl 11: 157-165, 2009.
5. Sabnis AS, Reilly CA, Veranth JM and Yost GS: Increased transcription of cytokine genes in human lung epithelial cells through activation of a TRPM8 variant by cold temperatures. Am J Physiol Lung Cell Mol Physiol 295: L194-L200, 2008.

6. Sabnis AS, Shadid M, Yost GS and Reilly CA: Human lung epithelial cells express a functional cold-sensing TRPM8 variant. Am J Respir Cell Mol Biol 39: 466-474, 2008.

7. Bidaux G,Flourakis M,Thebault S, et al:Prostate cell differentiation status determines transient receptor potential melastatin member 8 channel subcellular localization and function. J Clin Invest 117: 1647-1657, 2007.

8. Bidaux G, Beck B, Zholos A, et al: Regulation of activity of transient receptor potential melastatin 8 (TRPM8) channel by its short isoforms. J Biol Chem 287: 2948-2962, 2012.

9. Fernández JA, Skryma R, Bidaux G, et al: Short isoforms of the cold receptor TRPM8 inhibit channel gating by mimicking heat action rather than chemical inhibitors. J Biol Chem 287: 2963-2970, 2012.

10. Liu XL, Peng CW, Chen C, Yang XQ, Hu MB, Xia HS, Liu SP, Pang DW and Li Y et al: Quantum dots-based double-color imaging of HER2 positive breast cancer invasion. Biochem Biophys Res Commun 409: 577-582, 2011.

11. Meng F, Henson R, Wehbe-Janek H, Ghoshal K, Jacob ST, and Patel T: MicroRNA-21 regulates expression of the PTEN tumor suppressor gene in human hepatocellular cancer. Gastroenterology 133: 647-658, 2007.

12. Kim EK and Choi EJ: Compromised MAPK signaling in human diseases: An update. Arch Toxicol: Feb 18, 2015 (Epub ahead of print).

13. Frühwald J, Camacho Londoño J, Dembla S, et al: Alternative splicing of a protein domain indispensable for function of transient receptor potential melastatin 3 (TRPM3) ion channels. J Biol Chem 287: 36663-36672, 2012.

14. Chu X, Tong Q, Wozney J, et al: Identification of an N-terminal TRPC2 splice variant which inhibits calcium influx. Cell Calcium 37: 173-182, 2005.

15. Goldar S, Khaniani MS, Derakhshan SM, and Baradaran B: Molecular Mechanisms of Apoptosis and Roles in Cancer Development and Treatment. Asian Pac J Cancer Prev 16: 2129-2144, 2015

16. Schütz A, Schneidenbach D, Aust G, Tannapfel A, Steinert M and Wittekind C: Differential expression and activity status of MMP-1, MMP-2 and MMP-9 in tumor and stromal cells of squamous cell carcinomas of the lung. Tumour Biol 23: 179-184, 2002.

17. Yudin Y and Rohacs T: Regulation of TRPM8 channel activity. Mol Cell Endocrinol 353: 68-74, 2012.

18. Rodríguez-Berriguete G, Fraile B, Martínez-Onsurbe P, Olmedilla G, Paniagua R and Royuela M: MAP Kinases and Prostate Cancer. J Signal Transduct 2012: 169170, 2012. 